\title{
Finanzierungstrends mittelständischer Konzerne auf Basis der Konzernrechnungslegung - eine kritische Analyse am Beispiel der Region Osnabrück
}

\author{
Gunther Meeh-Bunse'1, Martin Welling \\ 1 Prof. dr, Hoschschule Osnabrueck \\ e-mail: g.meeh-bunse@hs-osnabrueck.de
}

SCHLÜSSElwörter KMU, Finanzierung, Finanzierungsmethoden, finanzielle Bedürfnisse

ZUSAMmenfassung Zweck - ist es herauszufinden, wie sich mittelständische Konzerne finanzieren.

Methode - Um diese Frage zu beantworten werden die Konzernabschlüsse von acht mittelständischen Unternehmen aus der Wirtschaftsregion Osnabrück auf ihre Finanzierungsarten hin untersucht. Um einen Vergleich zwischen Literatur und den festgestellten genutzten Finanzierungsarten herzustellen, wird vorab eine Literaturrecherche durchgeführt. Anschließend werden mögliche Finanzierungstrends aufgezeigt.

Ergebnisse - Die Innenfinanzierung ist weiterhin die wichtigste Finanzierungsart für mittelständische Konzerne. Sie wollen unabhängiger von Banken werden und meiden innovative Finanzierungsarten wie Sale-and-Lease-back oder Factoring. Der Bankkredit ist dennoch weiterhin die häufigste Art der Außenfinanzierung. Gegenüber ihren Gesellschaftern bestehen oft starke Abhängigkeiten. Leasing gewinnt mehr an Bedeutung.

Wertschöpfung - Aufgezeigt wird die typische Finanzierungsstruktur innerhalb einer Region, was als Basis für weitere Forschungsarbeiten z.B. im Vergleich zu anderen Regionen genutzt werden kann. Gleichzeitig finden sich Anhaltspunkte, inwieweit sich moderne Finanzierungsformen durchgesetzt haben.

\section{Group accounting - a critical analysis on the example of the Osnabrück region}

KEYWORDS medium-sized groups, financing, financing methods, financial needs, medium-sized companies

ABSTRACT Purpose - This paper examines how medium-sized groups satisfy their financial needs.

Research methodology - Therefore the annual financial statements of eight groups in the business region around Osnabrück were examined whether the used methods of financing cover the methods stated in the common literature. On this basis financing trends are deducted.

Findings - It was found out that medium-sized groups try to stay independent and avoid risks. They are more connected with partners. Internal financing is the most popular source of financing. Revenues generated by deliveries, services and gains on securities are the main components of internal financing. The bank loan is the dominating type of external financing. Leasing is increasing its popularity as an alternative financial solution. Medium-sized groups are trying to prevent 
dependency on banks and prefer internal financing methods. They avoid innovative financial instruments such as crowd funding, sale-and-leaseback or factoring.

Originality/value - The typical finance structure in a region is identified which can be used for further studies as a comparison with other regions. Simultaneously an indication for the degree of penetration of new forms of financing is recognized.

\section{Einführung}

Deutschlands mittelständische Unternehmen stehen vor großen Aufgaben. Die zunehmende Konkurrenz, die Finanzkrise und die verschärften Regeln von Basel II und Basel III beeinflussen das Handeln der Mittelständler. Für alle bevorstehenden Aufgaben ist Kapital notwendig. Doch woher bekommen Mittelständler Kapital? Das Ziel dieses Aufsatzes ist es herauszufinden, wie sich mittelständische Konzerne in der Region Osnabrück finanzieren. Vorab werden die in der „klassischen“ Literatur erwähnten Innen- und Außenfinanzierungsarten vorgestellt. Die Ergebnisse aus der Untersuchung von acht Konzernen werden anschließend kurz vorgestellt. Ein Soll/Ist-Vergleich sorgt für einen Abgleich der Literatur mit den Ergebnissen der Untersuchung. Auf dieser Basis werden Finanzierungstrends mittelständischer Unternehmen in der Region abgeleitet und die Ergebnisse in einem Fazit festgehalten.

Alle acht untersuchten Unternehmen stammen aus der Wirtschaftsregion Osnabrück. Unter mittelständischen Unternehmen werden inhabergeführte und nicht kapitalmarktorientierte Unternehmen gefasst. Um ein branchenübergreifendes Bild wiederzugeben, wurden Unternehmen aus unterschiedlichen Wirtschaftszweigen ausgewählt. Die Konzernabschlüsse beziehen sich auf das Berichtjahr 2013.

Ein Konzern besteht aus rechtlich selbstständigen Unternehmen, deren Entscheidungs- und Handlungsfähigkeit bei einem Mutterunternehmen liegt. Das Mutterunternehmen übt einen beherrschenden Einfluss auf ein oder mehrere Tochterunternehmen aus. Da die wirtschaftliche Handlungsfähigkeit beim Mutterunternehmen liegt, wird der Konzern in der Konzernrechnungslegung fiktiv als eine rechtliche Einheit betrachtet (Vgl. Busse von Colbe, 2011, S.535). Der Einfluss auf Entscheidungen der Töchter basiert entweder auf Regelungen bezüglich der Mehrheit der Stimmen am gezeichneten Kapital, einem Beherrschungsvertrag oder der Unternehmenssatzung. Ist das Mutterunternehmen eine Kapital- oder gleichgestellte Personengesellschaft und übt sie beherrschenden Einfluss auf ihre Tochterunternehmen aus, so muss grundsätzlich ein Konzernabschluss angefertigt werden (Vgl. Lüdenbach/Hoffmann, 2016, §290Rz.1). Zu den acht Konzernen gehören:

1. Amazonen-Werke H. Dreyer GmbH \& Co. KG (diverse Landmaschinen).

2. AVO-Werke August Beisse GmbH (Herstellung und Vertrieb von Gewürzmischungen, Zusatzstoffen, Marinaden).

3. Bohnenkamp-Verwaltungs-GmbH (Reifen, Räder, Fahrzeugbauteile).

4. b u w Holding GmbH (Kundenmanagement-Lösungen).

5. Felix Schoeller Holding GmbH \& Co. KG (Spezialpapierherstellung).

6. Hans-Heiner Müller Verwaltungs und Beteiligungs GmbH (Wessels \& Müller) (Großund Einzelhandel für Kfz-Teile, -Werkzeuge, -Maschinen). 
7. HomeStead GmbH \& Co KG (Tief-, Hoch- und Schlüsselfertig, Tunnelbau).

8. Meyer \& Meyer Holding GmbH \& Co. KG (Spediteur, Logistiker).

\section{Finanzierungsarten mittelständischer Unternehmen}

Finanzierungsarten können in Innenfinanzierung und Außenfinanzierung gegliedert werden. Bei der Außenfinanzierung investieren Kapitalgeber außerhalb des Unternehmens Geld in das Unternehmen. Bei der Innenfinanzierung wird Geld innerhalb des Unternehmens beschafft (Vgl. Busse von Colbe, 2011, S.388ff.). Hierbei überwiegt stark der Anteil der Innenfinanzierung gegenüber der Außenfinanzierung.

Tabelle 1. Innen- und Außenfinanzierung deutscher Unternehmen

\begin{tabular}{|l|c|c|}
\hline \multicolumn{1}{|c|}{ In Mrd. $€$} & 2012 & 2013 \\
\hline Innenfinanzierung & 180,7 & 194,5 \\
\hline Außenfinanzierung & 85,4 & 71,0 \\
\hline Insgesamt & 266,2 & 265,5 \\
\hline
\end{tabular}

Quelle: Deutsche Bundesbank (2014), S. 44.

$\mathrm{Zu}$ den am häufigsten verwendeten Mittel der internen Finanzierung gehört die Finanzierung aus dem Wertschöpfungsprozess, der Vermögensumschichtung und der Nutzung von Abschreibungen oder Rückstellungen.

Beim Wertschöpfungsprozess werden die Kosten durch den Verkauf von Gütern und Dienstleistungen gedeckt. Übersteigen die Erträge die Aufwendungen wird Gewinn erwirtschaftet. Bleibt dieser Gewinn im Unternehmen steigt das Eigenkapital des Unternehmens (Vgl. Busse von Colbe, 2011, S. 391f.).

Bei der Vermögensumschichtung wird Betriebsvermögen freigesetzt. Eine Möglichkeit ist der Verkauf von Vermögensgegenständen. Eine weitere Möglichkeit stellen Rationalisierungsmaßnahmen dar. Werden Bestände im Lager verkleinert oder wird Personal abgebaut, verringern sich die Auszahlungsverpflichtungen des Unternehmens. Weitere Möglichkeiten stellen das Sale-and-Lease-back-Verfahren und das Working Capital Management dar (Vgl. Busse von Colbe, 2011, S. 391).

Vermögensgegenstände werden über ihre Nutzungsdauer abgeschrieben. Abschreibungen können zur internen Finanzierung verwendet werden, da sie den Gewinn mindern, aber keine auszahlungsrelevanten Vorgänge auslösen. Das verfügbare Kapital kann zur Finanzierung genutzt werden, wohingegen Rücklagen für den Erwerb von neuem Betriebsvermögen getätigt werden sollten (Vgl. Wöhe, 2013, S. 431ff.).

Die Innenfinanzierung bietet für nicht kapitalmarktorientierte Unternehmen häufig die einzige Möglichkeit ihr Eigenkapital zu erhöhen. Die im Resultat höhere Eigenkapitalquote erleichtert die Fremdkapitalbeschaffung (Vgl. Busse von Colbe, 2011, S. 392).

Bei der Fremdfinanzierung als Teil Außenfinanzierung überlässt der Gläubiger dem Unternehmen schuldrechtlich Kapital auf Zeit ohne Eigentum zu erhalten. Folglich entstehen keine mittelbaren Mitsprache-, Entscheidungs- oder Kontrollrechte am Unternehmen. Es wird im 
Folgenden zwischen kurzfristigen und langfristigen Fremdkapital unterschieden. Kurzfristige Verbindlichkeiten weisen eine Laufzeit von 90 - 365 Tagen auf. Langfristiges Fremdkapital weist eine Mindestdauer von fünf Jahren auf (Vgl. Wöhe, 2013, S. 217ff.).

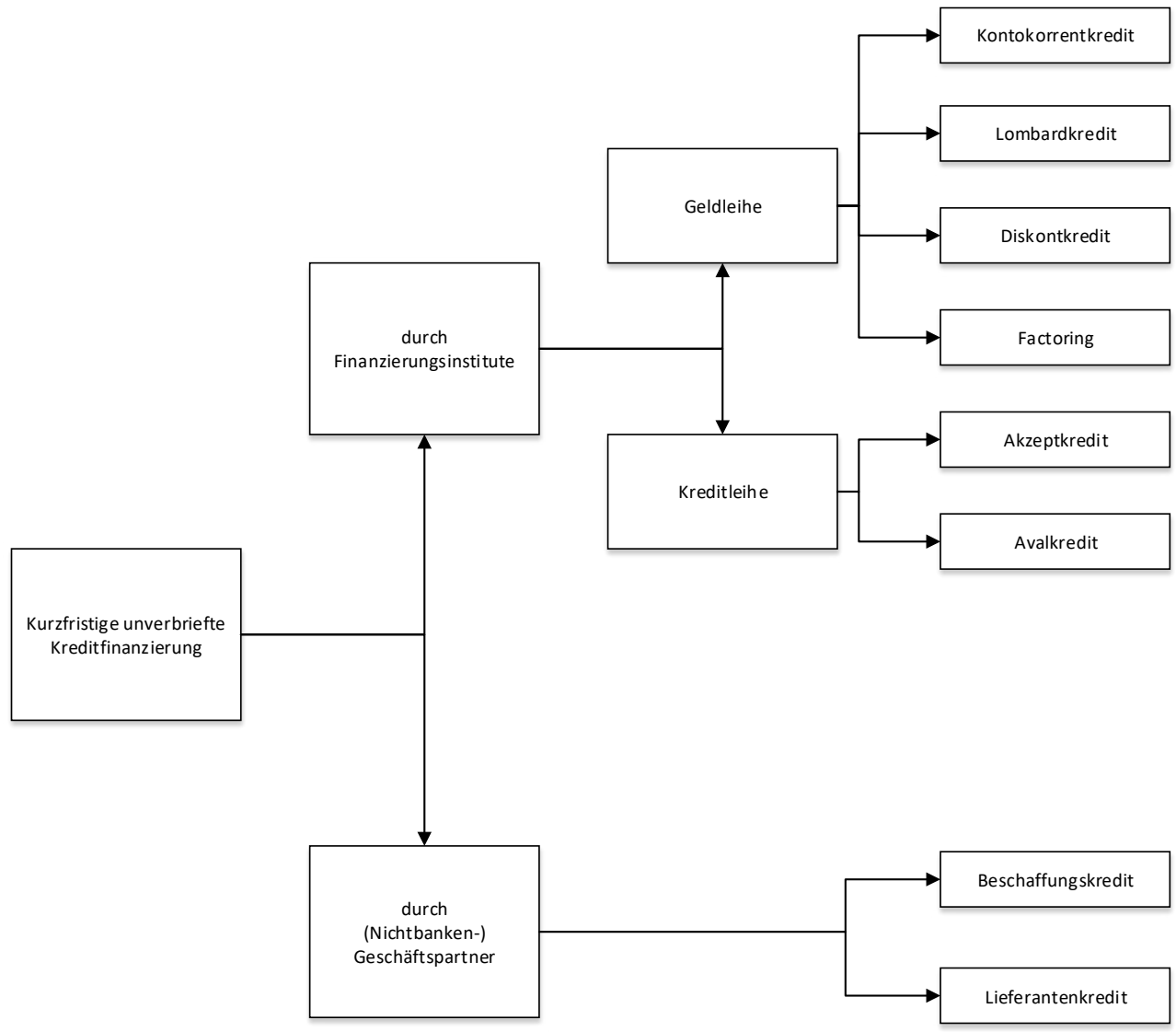

Abbildung 1. Formen der kurzfristigen Kreditfinanzierung

Quelle: Busse von Colbe (2011), S. 413.

Bei der Fremdfinanzierung wird unterschieden, ob die Finanzierung über ein Finanzinstitut oder durch einen Geschäftspartner erfolgt. Geschäftspartner sind Kunden und Lieferanten. Durch einen Lieferantenkredit wird dem Unternehmen eine längere Zahlungsfrist eingeräumt. Das Unternehmen kann Käufe tätigen ohne Bankkredite aufzunehmen.

Bei einer Vorauszahlung erhält das Unternehmen Zahlungen des Kunden für einen Auftrag im Voraus. Kosten sind hierdurch gedeckt ohne in Vorleistung zu gehen.

Kurzfristiges Fremdkapital, dessen Bereitstellung durch Finanzinstitute erfolgt, wird in drei Varianten eingeteilt. Die am häufigsten verbreitete Variante ist der Kontokorrentkredit. Das Konto bei der Bank darf bis zu einer gewissen Grenze überzogen werden, um kurzfristige Liquiditätsengpässe zu überbrücken (Vgl. Bitz, 2008, S. 65). 
Eine weitere Möglichkeit bietet das Factoring. Forderungen werden an das Factoring-Unternehmen abgetreten. Im Gegenzug erfolgt die Zahlung unmittelbar und nicht erst bei Fälligkeit der Zahlung. Das Forderungsausfallrisiko und das Mahnwesen kann mit abgetreten werden. Diese beiden Varianten gehören der Geldleihe an.

Der Avalkredit ist eine Form der Kreditleihe. Im Gegensatz zur Geldleihe erfolgt kein Zahlungseingang. Die Bank übernimmt Ausfallgarantien in einer festgeschriebenen Höhe, wodurch sich die Bonität des Unternehmens gegenüber den Gläubigern verbessert. Durch die gestiegene Bonität werden neue Kreditformen zugänglich.

Im Folgenden werden drei relevante Formen der langfristigen Kreditfinanzierung vorgestellt.

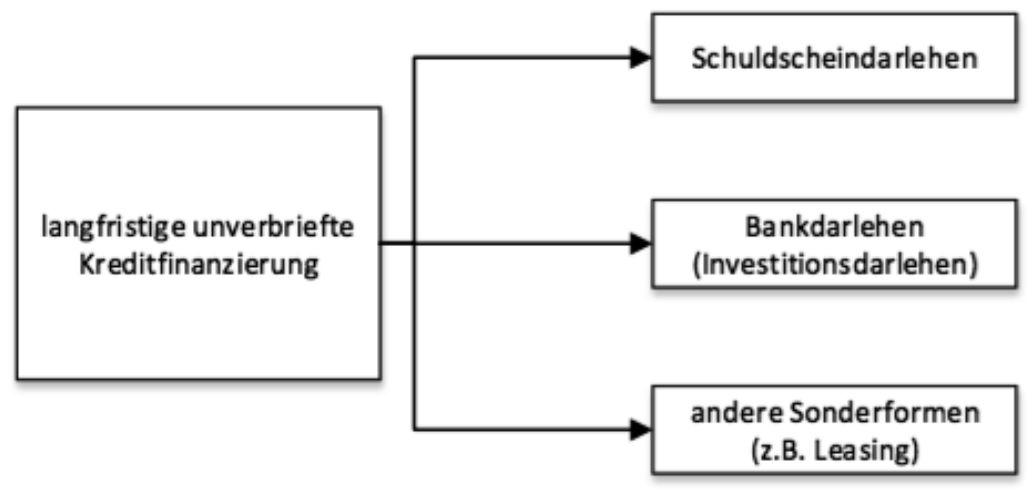

Abbildung 2. Formen der langfristigen Kreditfinanzierung

Quelle: Busse von Colbe (2011), S. 409.

Beim Darlehen wird der Darlehensgeber durch den Darlehensvertrag dazu verpflichtet dem Darlehensnehmer den im Vertrag genannten Betrag zur Verfügung zu stellen. Der Darlehensnehmer ist verpflichtet einen im Vertrag festgeschriebenen Zins zu zahlen und am Ende der Laufzeit den Darlehensbetrag zurückzuzahlen (Vgl. Wöhe, 2013, S. 247). Aufgrund gesetzlicher Richtlinien sind Banken verpflichtet eine interne Bonitätsprüfung über den Darlehensnehmer durchzuführen. Die verschärften Gesetze führen dazu, dass mittelständische Unternehmen Schwierigkeiten bei der Vergabe von langfristigen Bankdarlehen bekommen (Vgl. Busse von Colbe, 2011, S. 411f.).

Ein Schuldscheindarlehen ist ein anleiheähnlicher, allerdings nicht kapitalmarktorientierter Großkredit mit einer gewöhnlichen Laufzeit von zehn bis fünfzehn Jahren. Der Schuldschein bestätigt, dass der Darlehensbetrag dem Schuldner übergeben wurde. Die Beweislast wechselt vom Gläubiger auf den Schuldner, der Schuldschein stellt aber keine Beweisurkunde dar. Oft fungiert ein Kreditinstitut als Mittler, um die Schuldscheine treuhänderisch einzusammeln (Vgl. Ekkenga, 2014, S. 751).

Eine weitere Form ist das Leasing. Leasing wird oft aus bilanz- und steuerrechtlichen Aspekten gewählt. Hierbei werden dem Leasingnehmer, über eine vorher definierte Zeitdauer, vom Leasinggeber ein oder mehrere Objekte zur Verfügung gestellt. Der Leasingnehmer zahlt einen monatlichen Betrag an die Leasinggesellschaft. Instandhaltungskosten werden vom Leasingnehmer getragen. Eine Kündigungsmöglichkeit besteht häufig nicht. Neben dem Leasing 
ist die Miete eine weit verbreitete Finanzierungsmöglichkeit. Das Prinzip ähnelt dem Leasing, wohingegen der Vermieter für Instandhaltungskosten zuständig ist und üblicherweise Kündigungsmöglichkeiten vertraglich festgehalten sind (Vgl. Wirtschaftskammer Österreich, 2016).

$\mathrm{Zu}$ den alternativen Finanzierungsmöglichkeiten gehört unter anderem das Crowdfunding (Schwarmfinanzierung). Unternehmen oder Personen stellen über das Internet Projekte vor, die dann von Kapitalgebern finanziert werden können. Die Crowdfundingportale stellen die Plattform und verlangen hingegen eine Gebühr oder Provision. Vor allem Start-Ups und Einzelpersonen nutzen das Crowdinvesting. Mittlerweile nutzen einige mittelständische Unternehmen diese alternative Finanzierungsmöglichkeit (Vgl. Schwethelm, 2014, S. 4).

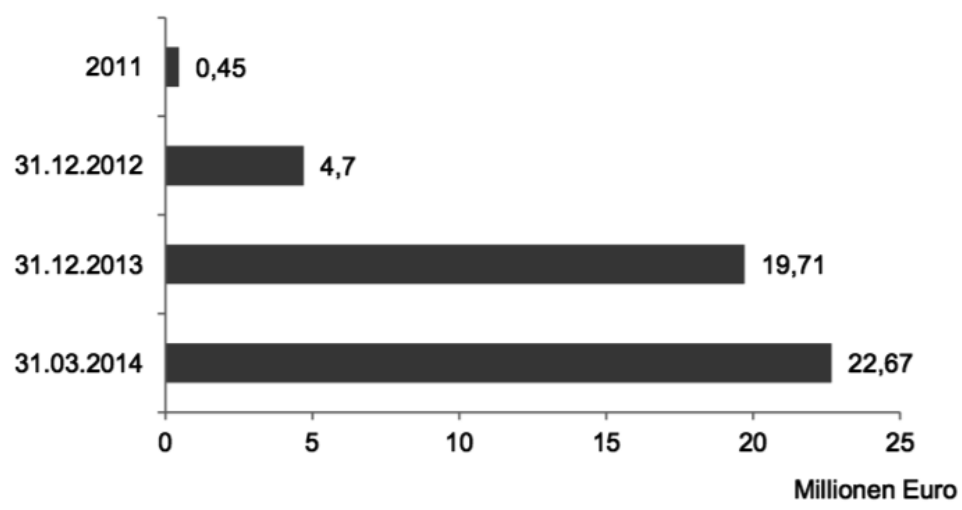

Abbildung 3. Entwicklung Crowdinvesting-Volumen

Quelle: Schwethelm (2014), S. 9.

Das Finetrading wird bisher nur von wenigen Mittelständlern genutzt. Ein Zwischenhändler (Finetrader) finanziert dem Kunden die Bestellung und zahlt dem Lieferanten direkt den Preis. Der Kunde zahlt den Wert zuzüglich Zinsen innerhalb von 120 Tagen zurück. Sofern Skonto genutzt werden kann, bietet Finetrading eine günstige Alternative (Vgl. Erben, 2009).

\section{Finanzierungsarten laut den Konzernabschlüssen}

Aus den Untersuchungen der Jahresabschlüsse wurden die vorgestellten Finanzierungsarten herausgefiltert. Die Höhe oder Anzahl der verwendeten Mittel ist kein Kriterium. Dabei wurde die Höhe und Anzahl nicht als Kriterium herangezogen, um den doch unterschiedlich großen mittelständischen Konzernen gerecht zu werden. Die Auswertung hätte dadurch verfälscht werden können und so in eine bestimmte Richtung gelenkt werden können. Sofern die Finanzierungsart genutzt wird, bildet sie einen Bestandteil der nachfolgenden Abbildung.

Wie zu erkennen ist, tauchen viele der bereits vorgestellten Finanzierungsmöglichkeiten in der Untersuchung auf. Die mit dominierende Finanzierungsart Gesellschafterdarlehen sowie die Finanzierungsarten Wertpapiere, Stille Beteiligung und Forderungen gegenüber Gesellschaftern wurden durch die klassische Literatur weniger in den Vordergrund vorgestellt. 


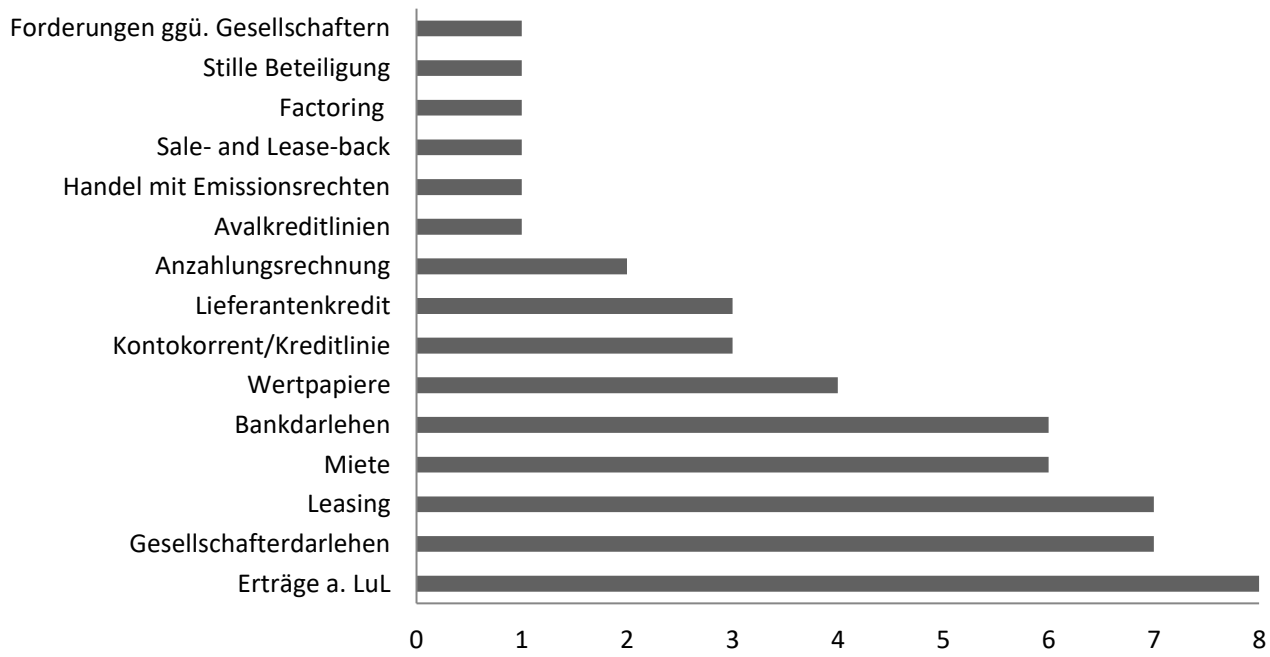

Abbildung 4. Absolute Häufigkeit der Finanzierungsarten

\section{Vergleich zwischen Literatur und Praxis}

Erträge aus Lieferungen und Leistungen sind, wie bereits vorgestellt, die primäre Finanzierungsquelle nicht-kapitalmarktorientierter Konzerne. Das Leasing und die Miete fanden ähnliche Beliebtheit. Das Bankdarlehen ist noch immer eines der am häufigsten genutzten langfristigen Finanzierungsmittel. Der Kontokorrentkredit bzw. die Kreditlinie sichert Unternehmen die kurzfristige Liquidität. Beliebte Finanzierungsmittel, die weniger in der klassischen Literatur auftauchten, sind das Gesellschafterdarlehen und Erträge aus Wertpapieren. Vorgestellte Finanzierungsmittel wie der Avalkredit, der Handel mit Emissionsrechten, Sale-and-Lease-back oder das Factoring fanden wenig Verwendung in mittelständischen Konzernen. Die stille Beteiligung und Forderungen gegenüber Gesellschaftern wurden weniger in der klassischen Literatur vorgestellt und werden aufgrund ihres geringen Vorkommens vernachlässigt. Im Folgenden sollen die noch nicht vorgestellten Finanzierungsarten beleuchtet werden.

Eine stille Beteiligung liegt vor, wenn eine Vermögenseinlage durch einen Investor getätigt wird, jedoch kein Eigentumsanspruch an der Gesellschaft entsteht. Der Investor ist an den Gewinnen beteiligt. Da ein stiller Gesellschafter wenig Mitspracherechte erhält und frisches Kapital in das Unternehmen fließt, wird die stille Beteiligung von den Unternehmen durchaus geschätzt. Die Bilanzierung als Fremd- oder Eigenkapital hängt häufig von der Vertragsausgestaltung ab (Vgl. Grunow/Figgener, 2006, S. 104).

Durch die Position Forderungen ggü. Gesellschaftern kann sich Kapital aus dem Unternehmen durch die Zinseinnahmen finanzieren. Diese Möglichkeit kann zu einer schlechteren Bonitätsbewertung seitens der Kreditinstitute führen, da sie das wirtschaftliche Eigenkapital im Rahmen des Ratings schwächt (Vgl. Achleitner/Everling, 2007, S. 83f.).

Die Hälfte der untersuchten Konzerne nutzten die Erträge aus dem Kauf und Verkauf von Wertpapieren Dritter zur Finanzierung. Es ging den Unternehmen nicht um eigene Emissionen. 
Das Gesellschafterdarlehen ist ein Darlehen der Gesellschafter an das Unternehmen und wird in der Bilanz als Fremdkapital ausgewiesen. Anstatt weitere Gesellschaftereinlagen hinzuzuführen, können als Nebeneffekt durch ein Gesellschafterdarlehen Gewerbesteuerzahlungen eingespart werden. Üblicherweise wird das Darlehen zu einem niedrigeren Zinssatz als dem Marktzinssatz gewährt (Vgl. Alt/Kaschny, 2015, S. 69ff.). Es kann davon ausgegangen werden, dass durch die Gewährung von Gesellschafterdarlehen die Kreditwürdigkeit bei Dritten steigt (Vgl. Grunow/Figgener, 2006, S. 294). Da dem Unternehmen Fremdkapital und kein Eigenkapital in Form einer Gesellschaftereinlage zugeführt wird, sinkt die Eigenkapitalquote.

Aus der Literaturrecherche und den Untersuchungen sehen wir zwei Sachverhalte als bestätigt. Zum einen ist die Innenfinanzierung weiterhin die wichtigste Finanzierungsart bei mittelständischen Konzernen in der Region. Die untersuchten Unternehmen nutzten alle die Erträge aus den Lieferungen und Leistungen, um sich zu finanzieren. In einer Studie der Wirtschaftsprüfungsgesellschaft Deloitte wurde gleichlautend festgestellt, dass $82 \%$ aller mittelständischen Unternehmen auf die Innenfinanzierung setzten (Vgl. Deloitte, 2012, S. 24ff.). Zum anderen bleibt der Bankkredit weiterhin die am häufigsten gewählte externe Finanzierungsart mittelständischer Konzerne in der Region. Ungeachtet einer berichteten Skepsis gegen Bankkredite (Vgl. Kreditanstalt für Wiederaufbau, 2017, S. 15) nutzen mittelständische Konzerne weiterhin den Bankkredit zur externen Finanzierung. Allerdings nutzen zwei der Konzerne keine Bankdarlehen.

\section{Hypothesen über Finanzierungstrends mittelständischer Unternehmen}

Aus der Literaturrecherche und den Untersuchungen lassen sich die folgenden Finanzierungstrends in der Region Osnabrück ableiten.

„Mittelständische Konzerne in der Region wollen unabhängiger von Banken werden.“ Ungeachtet der weiterhin führenden Rolle des Bankkredits nimmt seine relative Bedeutung ab (Vgl. Deutscher Sparkassen- und Giroverband, 2017. S. 4). Aus den Untersuchungen geht hervor, dass zwei Konzerne komplett ohne Bankkredite auskommen. Generell werden Bankkredite auf mehrere Banken verteilt oder zurückgefahren (Vgl. Wirtschaftswoche, 2014).

„Mittelständische Konzerne in der Region halten nichts von innovativen Finanzierungsarten." Die Verwendung von innovativen Finanzierungsarten wie Crowdinvesting oder Finetrading konnte nicht festgestellt werden. $\mathrm{Zu}$ den innovativsten Arten gehörten Factoring oder Sale-and-Lease-back, welche jedoch nur bei einem Konzern ermittelt wurden.

„Die Finanzierung über Leasing und Miete gewinnt bei mittelständischen Konzernen in der Region immer mehr an Bedeutung." Sieben von acht untersuchten Unternehmen nutzten Leasing. Der Trend hin zum Leasen und Mieten von Objekten ist teilweise durch die gestiegenen Anforderungen der Banken durch Basel III zu begründen. Die erschwerte Kreditaufnahme treibt Unternehmen zum Leasing (Vgl. Spohr, 2014).

„Mittelständische Konzerne in der Region sind häufig sehr abhängig von ihren Gesellschaftern.“ Neben den Einlagen der Gesellschafter nutzen sieben der acht mittelständischen Unternehmen außerdem das Gesellschafterdarlehen. Die gesamte Darlehenshöhe variiert bei den untersuchten Unternehmen stark. 
„Finanzerträge aus Wertpapiere gewinnen als Finanzierungsart an Bedeutung.“ Diese Finanzierungsart muss noch weiter untersucht werden. Es kann vermutet werden, dass in der Niedrigzinsphase durch realisierte Kursgewinne höhere Renditen erwirtschaftet werden.

\section{Fazit}

Neben den in der klassischen Literatur herausgestellten Finanzierungsarten wurden weitere Arten bei den mittelständischen Konzernen der Region identifiziert. Vor allem die Finanzierung durch Wertpapiere und Gesellschafterdarlehen wurde bei den untersuchten Unternehmen häufig genutzt und bedarf daher einer genaueren Untersuchung. Wie wichtig die Gesellschafterdarlehen für die Unternehmen sind, sollte untersucht werden. Ebenso sollten die Beweggründe für die Finanzierung über Wertpapiererträge untersucht werden. Die Korrelation zwischen Finanzierungsarten und Marktzinssatz könnte aus strategischen Gründen interessant sein. Es konnte herausgefunden werden, dass mittelständische Konzerne die verschärften Regelungen durch Basel III ernst nehmen und Alternativen suchen. Diese Alternativen sind momentan noch nicht die innovativen Finanzierungsmittel am Markt wie Crowdfunding oder Finetrading. Unter Umständen wäre es ratsam, dass innovative Finanzierungsmethoden mehr in Betracht gezogen werden, um in Zukunft weiterhin gut aufgestellt zu sein. Insgesamt nutzen die untersuchten Unternehmen eher risikoarme Finanzierungsarten. Diese Erkenntnisse und die generell gute Aufstellung der untersuchten Unternehmen, geben eine solide Basis für die Wirtschaftsregion Osnabrück.

\section{Literatur}

Achleitner, A., Everling, O. (2007). Finanzrating: Gestaltungsmöglichkeiten zur Verbesserung der Bonität. Wiesbaden: Gabler Verlag.

Alt, J., Kaschny, M. (2015). Alternative Finanzierungsformen im Mittelstand. Lohmar: Josef Eul Verlag.

Bitz, M., Stark, G. (2009). Finanzdienstleistungen. München: Oldenbourg Wissenschaftsverlag.

Busse von Colbe, W. et al. (2011). Betriebswirtschaft für Führungskräfte. Stuttgart: Schäffer-Poeschel.

Deloitte \& Touch GmbH Wirtschaftsprüfungsgesellschaft (2012). Studie: Finanzierung im Mittelstand. Heruntergeladen von:_https://www2.deloitte.com/content/dam/Deloitte/de/Documents/Mittelstand/Finanzierung-im-Mittelstand.pdf (14.01.2017).

Deutsche Bundesbank (2014). Monatsbericht Dezember 2014. Frankfurt a.M.: Verlag der Deutschen Bundesbank.

Deutscher Sparkassen- und Giroverband (2017). Diagnose Mittelstand 2017. Berlin. Heruntergeladen von: https://www. dsgv.de/de/presse/diagnose_mittelstand_2017.html (30.10.2017).

Ekkenga, J., Schröer, H. (2014). Handbuch der AG-Finanzierung. Köln: Karl Heymanns Verlag.

Erben, S. (2009). Finetrading - Vorschuss vom Zwischenhändler. Heruntergeladen von: http://www.handelsblatt.com/ unternehmen/mittelstand/finetrading-vorschuss-vom-zwischenhaendler/3318816.html (14.01.2017).

Grunow, H., Figgener, S. (2006). Handbuch moderne Unternehmensfinanzierung: Strategien zur Kapitalbeschaffung und Bilanzoptimierung. Berlin: Springer-Verlag.

Kreditanstalt für Wiederaufbau (2017). KfW-Mittelstandspanel 2017, Frankfurt: Heruntergeladen von: pöühttps:// www.kfw.de/KfW-Konzern/KfW-Research/KfW-Mittelstandspanel.html (9.11.2017).

Hoffmann,W.-D./Lüdenbach, N. (2016). NWB-Kommentar Bilanzierung. Herne: NWB-Verlag.

Schwethelm, C. (2014). Crowdinvesting als Finanzierungsalternative für mittelständische Unternehmen. Göttingen: VWA Göttingen.

Spohr, F. (2014). Handelsblatt: Alternativen zum Kredit der Hausbank-Maschine zur Miete. 
Wirtschaftskammer Österreich (Hrsg.) (2016). Leasing - Eine Alternative zum Kredit. Heruntergeladen von: https:// www.gruenderservice.at/Content.Node/gruenden/Finanzierung-und-Foerderung/Alternative-Finanzierungen/ Leasing.html (14.01.2017).

Wirtschaftswoche (Hrsg.) (2014). Unternehmensfinanzierung Mittelständler pfeifen auf Bankkredite. Abrufbar unter: http:/www.wiwo.de/unternehmen/mittelstand/unternehmensfinanzierung-mittelstaendler-pfeifen-auf-bank kredite-/10057268.html (4.01.2017).

Wöhe, G. et al. (2013). Grundzüge der Unternehmensfinanzierung. München: Vahlen.

\section{Cytowanie}

Meeh-Bunse, G., Welling, M. (2017). Finanzierungstrends Mittelständischer Konzerne auf Basis der Konzernrechnungslegung - Eine Kritische Analyse am Beispiel der Region Osnabrück. Współczesne Finanse. Teoria i Praktyka, 1 (2), 35-44. DOI: 10.18276/wf.2017.1-04. 